\title{
The interictal activities load and cognitive performance of children with typical absence epilepsy
}

\author{
Shereen Ahmed ELAhwal ${ }^{1}$, Yasser Abo Elfotoh El-Heneedy ${ }^{2}$, Wafik Said Bahnasy ${ }^{2^{*}}$ (D), \\ Reham Abdel Rahman Amer $^{2}$ and Khaled Hussein Rashed ${ }^{2}$
}

\begin{abstract}
Background: The description of childhood absence epilepsy (CAE) a benign self-limited generalized epilepsy has become a matter of debate. The objectives of this work were to evaluate the existence of psychiatric and cognitive impairments among patients with typical CAE and to correlate their possible relation to seizure frequency, duration of epilepsy, IISL, and valproate therapy.

Methods: The study was conducted on 19 typical CAE patients receiving valproate therapy, 11 newly diagnosed CAE patients not receiving AEDs, and 30 healthy control subjects (HCS). Participants were subjected to medical history taking, EEG monitoring, child behavior checklist (CBCL), Stanford Binet Intelligence Scale 5th edition, and computerized psychometric tests that assess cognitive domains and executive functions.

Results: The study revealed a high rate of cognitive and psychiatric dysfunctions in CAE patients. 53.3\% of patients had psychiatric problems versus $16.6 \%$ in HCS. Attention deficit hyperactive disorder (ADHD) (26.6\%), anxiety (16.6\%), and depression (6.6\%) were the most common psychiatric disorders in the patient group. Withdrawn/ depressed symptoms, thought problems, social problems, and attention problems in CAE patients were significantly increased compared to HCS. At the same time, CAE patients perform worse in cognitive scales than HCS with comparable intelligent quotient (IQ) scores.
\end{abstract}

Conclusion: Cognitive and psychiatric impairments in typical CAE patients appear multifactorial in origin with epilepsy-related factors including the duration of epilepsy and interictal spike load (IISL).

Keywords: Childhood absence epilepsy, Cognition, Interictal discharge load, Valproate

\section{Introduction}

Childhood absence epilepsy (CAE) is an idiopathic agerelated non-motor generalized epilepsy that usually begins between 3 and 12 years and is characterized by brief episodes of starring (10-20 s with an abrupt onset and offset), easily provoked by hyperventilation (HV), and is accompanied by impaired awareness, behavioral arrest, eyelid fluttering, or hand/face automatisms [1]. It

\footnotetext{
* Correspondence: wafiq.elbahnasi@med.tanta.edu.eg;

wafiq.elbahnasi@med.tanta.edu.eg

${ }^{2}$ Department of Neurology, Faculty of Medicine, Tanta University, Tanta 31527, Egypt

Full list of author information is available at the end of the article
}

is more common in girls than boys and constitutes about $10-15 \%$ of childhood-onset epilepsy with an estimated prevalence of $0.4-0.7$ per 1000 population [2]. Typical absence is characterized by the pathognomonic interictal $2.5-4 \mathrm{~Hz}$ generalized spike-wave discharges (SWD) on EEG which lasts 4-25 s [3].

The description of CAE as a benign disease had been given way because affected children often show various social, cognitive, emotional, and behavioral problems despite their appropriate response to antiepileptic drugs (AEDs) and good seizure control [4, 5]. There are controversial data about the nature of these cognitive or psychiatric impairments whether they are related to the 
epileptic syndrome, the AEDs, or a combination of both [6]. The interaction between the interictal spikes (IIS) and cognitive impairment is still a matter of research as the IIS may produce disturbances in neural processing called transitory cognitive impairment [7].

\section{Aim of the work}

Aims of the work were to evaluate the existence of psychiatric and cognitive impairments among patients with typical CAE and to correlate their possible relation to seizure frequency, duration of epilepsy, interictal spike load (IISL), and valproate therapy.

\section{Methods}

This study was an observational cross-sectional casecontrol study performed on 19 consecutive patients with typical CAE on valproate therapy, 11 newly diagnosed CAE patients not receiving AEDs, and 30 healthy control subjects (HCS) matching patients' age, sex, and educational level. Patients and HCS were selected from the epilepsy clinic in the Neurology and Psychiatry Center, Tanta University Hospitals, from the period 1 April 2017 to 31 March 2019. Typical CAE was diagnosed according to the revised criteria of the International League Against Epilepsy (ILAE) 2017 for absence epilepsy [8].

Exclusion criteria comprised of children with atypical absence epilepsy, intellectual disabilities, developmental delay, premorbid psychiatric problems, neuro-metabolic disorders, or those receiving drugs with a negative impact on cognition.

The study was approved by the local Ethical Research Committee of the Faculty of Medicine, Tanta University Hospitals. Informed consent was obtained from all subjects' guardians before participation in the study and any potential risks were clarified to them.

All participants were subjected to medical history taking, neurological examination, routine laboratory tests, measurement of serum valproic acid (in treated patients' group), and blood level of ammonia. They were also submitted to simultaneous long-term EEG monitoring and cognitive assessment. Long-term EEG for $2 \mathrm{~h}$ was done in the morning by two different epileptologists with experience of more than 15 years in EEG analysis. Comparison between both raters showed no interrater variability. Provocative tests like post hyperventilation and photic stimulation were used.

The mean IISL was calculated and correlated with the cognitive assessment results. EEG was visually rated by two different epileptologists. The number of spike-wave in $2 \mathrm{~h}$ was calculated and divided on 120 where the mean spike per minute was estimated for every case.

The EEG was performed using the Nihon Kohden EEG system (model EEG-1200, 64 channels, Japan) regarding the American Clinical Neurophysiology Society guidelines [9].

The IQ was measured by the Arabic validated version of Stanford Binet Intelligence Scale 5th ed. [10], and the cognitive functions were evaluated by applying the computerized version 0.14 of The Psychology Experiment Building Language (PEBL) and PEBL Test Battery [11]. We assessed the executive planning/set-shifting by the Wisconsin Card Sorting Test [12], the problem-solving by Tower of London test [13], the attention/response inhibition by Stroop test, the processing speed by Trail Making Test, the fine motor speed by finger tapping test, the complex motor control by pursuit rotor task, the working memory by Corsi's Block Tapping Test [11], and the language/verbal fluency by the Arabic version of the Controlled Oral Word Association Test (COWAT) [14].

All the psychometric tests were done in a single session by the psychiatrist in the morning after performing the specified laboratory investigation. Each psychometric test is explained in detail in the Arabic language to each participant before starting to apply the test battery.

A psychiatric evaluation was done using the Arabic translation of the Kiddie Schedule of Affective Disorders and Schizophrenia (K-SADS) [15]. The emotional and behavioral problems were assessed by using an Arabic validated form of the Child Behavior Checklist (CBCL) that was fulfilled by the guardians and scored by a computerized software system (Assessment Data Managerversion 9.1) [16].

Statistical presentation and analysis were conducted using the Statistical Program for Social Science (SPSS) Version 20.0, IBM, Armonk, NY, USA. Quantitative data were expressed as mean \pm standard deviation (SD), and qualitative data were expressed as frequency and percentage. Chi-square $\left(\chi^{2}\right)$ and ANOVA tests were also used.

\section{Results}

Demographic data of CAE patients and the control group revealed that their ages were $7.97 \pm 1.71$ years and $8.13 \pm 2.19$ years, respectively. Sixty percent were females $(18 / 30)$, and $40 \%$ were males $(12 / 30)$ in both groups. Twenty-eight (93.3\%) patients and 29 (96.6\%) in the control group were educated. Both groups were between kindergarten (KG1) to 4th year primary school grade. The studied groups were age-, educational level-, and sex-matched.

In the CAE group, the mean disease duration was $11.43 \pm 9.64$ months. Treated patients (19/30) 63.3\% receiving sodium valproate (VPA) in the dose range of 800-2000 mg/day and (11/30) 36.7\% were newly diagnosed. The average blood levels of VPA were within the therapeutic range of $82.21 \pm 16.46 \mathrm{mg} / \mathrm{dl}$. Sixty percent were seizure-free for at least 6 months based on parental 
reports, while others had ongoing seizures ranging from less than 1 to up to 20 seizures per day.

In CAE children, about 16 patients (53.3\%) had psychiatric disorders, 8 children had ADHD (26.6\%), 5 children had anxiety (16.6\%), 2 had depression disorder (6.6\%), and one child (3.3\%) had both depression and anxiety. However, in the control group, 5 participants (16.6\%) only had a psychiatric illness, 3 children with ADHD (10\%), 1 child with anxiety (3.3\%), and one child with both ADHD and depression.

The results of CBCL for our participants showed that there were significant increases in each of withdrawn/depressed symptoms, thought problems, attention problems, and social problems in both treated (group I) and newly diagnosed (group II) patients compared to controls (group III), while comparison between the treated and newly diagnosed patients showed non-significant difference. On the other hand, the somatic complaints, anxious/depressed symptoms, delinquent, and aggressive behaviors showed non-significant differences between the studied groups (Table 1).

Cognitive assessments of our studied groups showed that there were statistically significant differences in group I and group II compared to the control (group III). The cognitive impairment in CAE patients included deficits in attention, complex motor tasks, verbal fluency, and executive functions (problem-solving, executive planning, and set-shifting) as shown in Table 2 . Comparison between the treated and newly diagnosed patients showed non-significant difference except for Wisconsin Card Sorting Test (WCST).

Compared to the control subjects, 14 CAE patients (46.6\%) had language dysfunction, 19 patients (63.33\%) had attention problems, 13 patients (43.6\%) had impaired problem-solving, 10 patients $(33.33 \%)$ had problems in planning/set-shifting and 21 patients (70\%) had dysfunction in complex motor control.

There was no significant effect of epilepsy-related variables (age of onset, frequency of seizure) on these psychiatric and cognitive abnormalities as shown in Table 3, while disease duration and IISL were correlated with these abnormalities. The mean IISL was $1.43 \pm 1.78$ spike/min. There was a positive correlation between disease duration and psychiatric symptoms (thought problems) and a negative correlation between IISL and WCST as illustrated in Fig. 1.

\section{Discussion}

Childhood absence epilepsy not only affects subcortical structure but disrupts regions of the frontal cortex, cingulate, and thalamus as well. This may explain the affection of behavior and cognition in CAE. The question arises whether all these deficiencies are attributed to the disease itself or the use of AEDs [17].

In this study, there was a high rate of psychiatric dysfunctions among the CAE group with ADHD, anxiety, and depression representing the most encountered psychiatric disorders. These results agreed with Caplan et al. [18] who documented that $61 \%$ of the CAE group had a psychiatric diagnosis, especially anxiety disorders and ADHD. In the same line, Vega et al. [19] found that CAE increased the patients' risk for affective problems, including anxiety and depression. From the biological point of view, the frontal lobe, thalamus, and serotonin dysfunction are concerned with both anxiety disorders and absence of epilepsy [20].

CAE patients perform worse in cognitive tasks than their healthy counterparts despite having IQ levels within the average range. This cognitive impairment was significantly noticeable in attention, complex motor tasks, verbal fluency, and executive functions among the former group, which may be reflected in their academic achievements. These findings were following previous studies $[21,22]$ that documented that patients with CAE suffered problems in attention and executive functions.

Verrotti et al. [23] reported that the CAE has affected mainly verbal skills and executive functions and did not affect memory. Caplan et al. [18] found that $25 \%$ of

Table 1 Comparison between studied groups in child behavior checklist

\begin{tabular}{|c|c|c|c|c|c|c|}
\hline & \multirow{2}{*}{$\begin{array}{l}\text { Group I } \\
(n=19)\end{array}$} & \multirow{2}{*}{$\begin{array}{l}\text { Group II } \\
(n=11)\end{array}$} & \multirow{2}{*}{$\begin{array}{l}\text { Group III } \\
(n=30)\end{array}$} & \multicolumn{3}{|c|}{ ANOVA test ( $p$-value) } \\
\hline & & & & I vs. II & I vs. III & II vs. III \\
\hline Withdrawn & $59.89 \pm 5.29$ & $61.64 \pm 3.01$ & $52.80 \pm 2.73$ & 0.228 & $0.001^{*}$ & $0.001^{*}$ \\
\hline Social problem & $57.84 \pm 4.82$ & $60.73 \pm 4.22$ & $54.53 \pm 4.74$ & 0.109 & $0.019^{*}$ & $0.001^{*}$ \\
\hline Thought problem & $60.21 \pm 4.48$ & $58.91 \pm 5.89$ & $51.87 \pm 2.16$ & 0.376 & $0.001^{*}$ & $0.001^{*}$ \\
\hline Somatic complaint & $55.74 \pm 5.35$ & $54.18 \pm 3.63$ & $54.23 \pm 4.22$ & 0.367 & 0.261 & 0.974 \\
\hline Anxious/depressed & $54.89 \pm 4.24$ & $53.82 \pm 3.84$ & $54.23 \pm 2.78$ & 0.419 & 0.521 & 0.737 \\
\hline Attention & $59.89 \pm 5.38$ & $61.09 \pm 5.54$ & $52.23 \pm 3.02$ & 0.474 & $0.001^{*}$ & $0.001^{*}$ \\
\hline Aggressive & $54.16 \pm 3.86$ & $53.55 \pm 3.21$ & $53.17 \pm 3.43$ & 0.649 & 0.343 & 0.762 \\
\hline Delinquent & $52.26 \pm 3.46$ & $52.09 \pm 1.04$ & $51.93 \pm 2.21$ & 0.859 & 0.660 & 0.861 \\
\hline
\end{tabular}

*Significant 
Table 2 Comparison between studied groups in cognitive tests

\begin{tabular}{|c|c|c|c|c|c|c|}
\hline & Group I & Group II & Group III & ANOVA & (p-value) & \\
\hline & & & & I vs. II & I vs. III & II vs. III \\
\hline Intelligence quotient & $87.58 \pm 7.51$ & $93.73 \pm 9.09$ & $91.77 \pm 8.97$ & 0.063 & 0.101 & 0.518 \\
\hline Finger tapping (DH) & $104.26 \pm 4.65$ & $105.64 \pm 5.59$ & $105.83 \pm 5.13$ & 0.478 & 0.295 & 0.913 \\
\hline Finger tapping (NDH) & $104.79 \pm 4.57$ & $102.55 \pm 2.84$ & $105.67 \pm 4.37$ & 0.165 & 0.480 & 0.056 \\
\hline COWAT & $92.68 \pm 2.54$ & $99.55 \pm 10.54$ & $110.90 \pm 10.80$ & 0.059 & $0.001^{*}$ & $0.001^{*}$ \\
\hline Stroop (s) & $163.89 \pm 21.86$ & $169.55 \pm 22.76$ & $129.17 \pm 12.52$ & 0.409 & $0.001^{*}$ & $0.001^{*}$ \\
\hline Trail making A (s) & $61.79 \pm 12.70$ & $59.55 \pm 12.48$ & $56.63 \pm 14.11$ & 0.660 & 0.195 & 0.540 \\
\hline Trail making B (s) & $124.89 \pm 61.66$ & $149.36 \pm 43.66$ & $125.30 \pm 45.66$ & 0.210 & 0.978 & 0.186 \\
\hline Corsi's block tapping test & $4.37 \pm 0.81$ & $4.53 \pm 0.72$ & $4.52 \pm 0.74$ & 0.583 & 0.498 & 0.978 \\
\hline Tower of London (correct moves) & $82.16 \pm 10.80$ & $84.18 \pm 12.45$ & $95.07 \pm 9.12$ & 0.607 & $0.001^{*}$ & $0.004^{*}$ \\
\hline WCST (conceptual level response) & $43.53 \pm 6.73$ & $49.73 \pm 8.00$ & $54.60 \pm 6.05$ & $0.017^{*}$ & $0.001^{*}$ & $0.042^{*}$ \\
\hline Pursuit rotor task (s) & $35.95 \pm 7.74$ & $37.36 \pm 6.10$ & $20.13 \pm 8.23$ & 0.631 & $0.001^{*}$ & $0.001^{*}$ \\
\hline
\end{tabular}

*Significant

COWAT controlled oral word association test, $D H$ dominant hand, NDH non-dominant hand, WCST Wisconsin Card Sorting Test

children with CAE had subtle cognitive dysfunction and 43\% had linguistic problems, while Vindhiya et al. [24] have recognized significant language difficulties and impaired visual memory in their CAE patients. Cheng et al. [25] found deficits in visual attention, intelligence, and executive functions with normal language speed among CAE children. Dissimilar selection criteria and different assessment tools may explain the conflicting results between different series.

In the present study, the patients' age of onset and the rate of seizures did not significantly influence the cognitive performance, while the duration of epilepsy was considerably correlated with cognitive dysfunctions. Gerhard et al. [26] also stressed the adverse effects of a longer duration of epilepsy on the intellectual functioning of adults and children with epilepsy. Cormack et al. [27] stated that the onset of epilepsy is a risk factor for cognitive disabilities if it occurs in the first 3 years of life.
The AEDs diminish neuronal irritability; hence, it could lessen neuronal excitability and decrease cognitive functions. In this study, valproate seems not impairing cognitive ability when its dosage falls within the normal therapeutic range. Sirisamut et al. [28] also showed that the VPA group did not exhibit any significant differences to normal comparators; the most marked adverse effects that affect cognition were observed with barbiturates and benzodiazepines, while the cognitive side effects of phenytoin, valproate, and carbamazepine did not reach the significant difference.

In this study, the high IISL was correlated with cognitive impairment. These results agree with Fonseca et al. [29] who found that suppression of interictal epileptiform discharges (IEDs) is associated with improved cognitive functions in children with epilepsy. These results were also consistent with the outcomes of Ung et al. [30] who reported that frequent IEDs in more than $10 \%$ of the record can impair cognitive performance in children.

Table 3 Correlation between epilepsy-related factors with cognitive and psychiatric abnormalities

\begin{tabular}{|c|c|c|c|c|c|c|c|c|}
\hline & \multicolumn{2}{|c|}{ Duration of disease } & \multicolumn{2}{|c|}{ Frequency of seizure } & \multicolumn{2}{|c|}{ Age of onset } & \multicolumn{2}{|l|}{ IISL } \\
\hline & $r$ & $p$ & $r$ & $p$ & $r$ & $p$ & $r$ & $p$ \\
\hline Withdrawn & 0.485 & $0.011^{*}$ & -0.116 & 0.540 & 0.177 & 0.349 & 0.493 & $0.008^{*}$ \\
\hline Social problem & 0.305 & $0.092^{*}$ & 0.243 & 0.195 & -0.106 & 0.576 & 0.287 & 0.105 \\
\hline Thought problem & 0.512 & $0.001^{*}$ & 0.216 & 0.348 & 0.132 & 0.486 & 0.473 & $0.010^{*}$ \\
\hline Attention & 0.392 & $0.042^{*}$ & -0.047 & 0.806 & -0.027 & 0.886 & 0.597 & $0.001^{*}$ \\
\hline Stroop time in $\mathrm{s}$ & 0.513 & $0.001^{*}$ & 0.237 & 0.208 & 0.101 & 0.596 & 0.639 & $0.001^{*}$ \\
\hline Tower of London & -0.602 & $0.001^{*}$ & 0.214 & 0.257 & -0.093 & 0.624 & 0.719 & $0.001^{*}$ \\
\hline COWAT & -0.412 & $0.019^{*}$ & -0.356 & 0.053 & -.063 & 0.742 & -0.593 & $0.001^{*}$ \\
\hline WCST & -0.682 & $0.001^{*}$ & -.147 & 0.439 & -0.058 & 0.760 & -0.757 & $0.001^{*}$ \\
\hline Pursuit rotor task & 0.529 & $0.001^{*}$ & -0.023 & 0.903 & 0.228 & 0.225 & 0.487 & $0.009^{*}$ \\
\hline
\end{tabular}

*Significant 

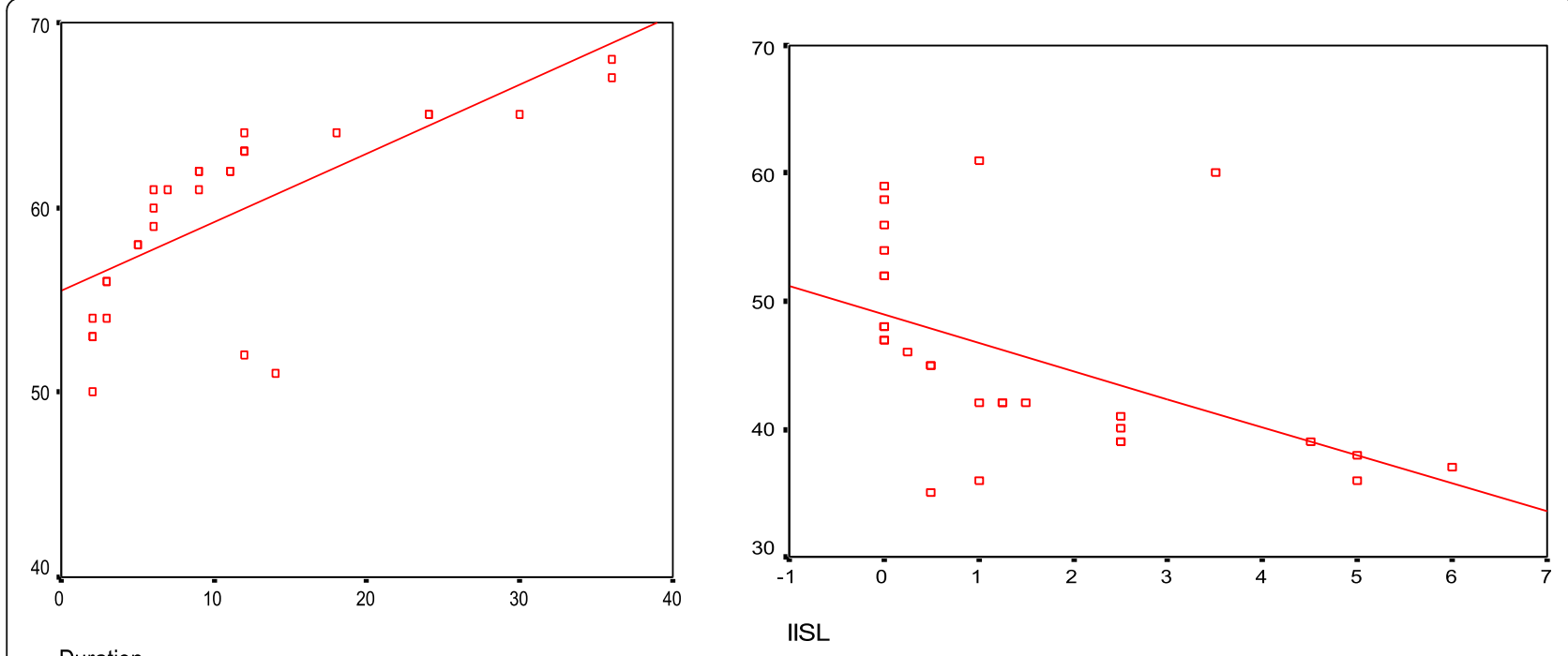

Duration

Fig. 1 Positive correlation between disease duration and thought problem (left), and a negative correlation between IISL and WCST (right)

\section{Conclusion}

The traditional descriptions of CAE as a benign disease had been given way. ADHD, anxiety, and depression represented the most encountered psychiatric disorders. The cognitive impairment was significantly noticeable in attention and executive functions. These psychiatric and cognitive abnormalities were correlated with disease duration and IISL.

\section{Recommendations}

Clinicians should be alert for early recognition and management of children suffering from CAE. This chiefly concerns children with extended duration of illness. Choosing AEDs, that pose high performance in controlling seizures and had negligible cognitive adverse effects, with gradual titration of these drugs and using them in their lowest effective dose is a very important issue. Follow-up of patients with EEG, even if they are seizure-free, for detection of IIS discharge is crucial because suppression of these troublesome discharges is associated with improved cognitive functions.

\section{Limitations}

A bigger sample size and double-blind study could increase the sensitivity and sensibility of the results.

\section{Abbreviations}

AEDs: Antiepileptic drugs; ADHD: Attention deficit hyperactive disorder; CAE: Childhood absence epilepsy; CBCL: Child behavior checklist; COWAT: Controlled oral word association test; HCS: Healthy control subjects; HV: Hyperventilation; IEDs: Interictal epileptiform discharges; IIS: Interictal spikes; IISL: Interictal spike load; ILAE: International League Against Epilepsy; IQ: Intelligent quotient; K-SADS: Kiddie Schedule for Affective Disorders and Schizophrenia; PEBL: Psychology Experiment Building Language; VPA: Sodium valproate; SWD: Spike-wave discharges; WCST: Wisconsin Card Sorting Test

\section{Acknowledgements}

We would like to thank the epilepsy clinic medical and para-medical staff, Neuropsychiatry Department, Tanta University Hospitals, for their great help in patients' selection and neurocognitive evaluation.

\section{Authors' contributions}

SAE participated in the study's design, patients' selection, EEG interpretation, statistical analysis, data analysis, references collection, manuscript writing, and final approval. YAH participated in study idea and design, patients' assessment and inclusion, EEG interpretation, statistical analysis, references collection, manuscript writing, and final approval. WSB participated in study idea and design, patients' assessment and inclusion, EEG interpretation, statistical analysis, references collection, manuscript writing, and final approval. RAA participated in study's design, patients' neurocognitive assessment, data analysis, manuscript revision, and final approval. KHR participated in the study's design, patients' selection, EEG interpretation, statistical analysis, data analysis, references collection, manuscript writing, and final approval.

\section{Funding}

No funding had been received.

\section{Availability of data and materials}

The datasets used and/or analyzed during the current study are available from the corresponding author on reasonable request.

\section{Declarations}

\section{Ethics approval and consent to participate}

- The manuscript was approved from The Research Ethics Committee and Quality Assurance Unit, Faculty of Medicine, Tanta University.

- The URL: http://tqac.tanta.edu.eg/new-tqac/

- QualityAssuranceUnit@hotmail.com

- Approval code: 31478/04/17

- Name of the PI: Wafik Said Bahnasy

- Name of the department: Neuropsychiatry

- Type of the research: promotion research

- Date of approval: April 2017

- The study's protocol had been permitted by The Research Ethics Committee and Quality Assurance Unit, Faculty of Medicine, Tanta University. Participations were voluntary, informed written consents were approved by participants' guardians, and any possible risks were clarified. 


\section{Consent for publication}

Not applicable.

\section{Competing interests}

The authors declare that they have no competing interests.

\section{Author details}

${ }^{1}$ Tanta University Hospitals, Tanta, Egypt. ${ }^{2}$ Department of Neurology, Faculty of Medicine, Tanta University, Tanta 31527, Egypt.

Received: 19 September 2020 Accepted: 21 March 2021

Published online: 10 May 2021

\section{References}

1. Morse E, Giblin K, Chung MH, Dohle C, Berg AT, Blumenfeld H. Historical trend toward an improved long-term outcome in childhood absence epilepsy. Epilepsy Res. 2019;152:7-10. https://doi.org/10.1016/j.eplepsyres.2 019.02.013.

2. Nikolić D, Marinković M, Međo B, Jovanović K. Absence epilepsy electroclinical features and current advances. Paediatrics Today. 2016;12(1): 131-8. https://doi.org/10.5457/p2005-114.149.

3. Aungaroon G, Arya R, Arthur TM, Holland KD. Ictal nose wiping in childhood absence epilepsy: a case series. Clin Neurol Neurosurg. 2018;172:134-6. https://doi.org/10.1016/j.clineuro.2018.06.035.

4. Verrotti A, Matricardi S, Rinaldi VE, Prezioso G, Coppola G. Neuropsychological impairment in childhood absence epilepsy: review of the literature. J Neurol Sci. 2015;359(1-2):59-66. https://doi.org/10.1016/j.jns.2 015.10.035.

5. Wang $X$, Jiao D, Zhang $X$, Lin X. Altered degree centrality in childhood absence epilepsy: a resting-state fMRI study. J. Neurol. Sci. 2017;373:274-9. https://doi.org/10.1016/j.jns.2016.12.054.

6. Kim EH, Ko TS. Cognitive impairment in childhood-onset epilepsy: up to date information about its causes. Korean J Pediatr. 2016;59(4):155-64. https://doi.org/10.3345/kjp.2016.59.4.155.

7. Loughman A, Bowden SC, D'Souza W. Cognitive functioning in idiopathic generalized epilepsies: a systematic review and meta-analysis. Neurosci Biobehav Rev. 2014;43:20-34. https://doi.org/10.1016/j. neubiorev.2014.02.012.

8. Fisher RS, Cross JH, D'Souza C, French JA, Haut SR, Higurashi N, et al. Instruction manual for the ILAE 2017 operational classification of seizure types. Epilepsia. 2017;58(4):531-42. https://doi.org/10.1111/epi.13671.

9. American Clinical Neurophysiology Society. Guideline twelve: guidelines for long-term monitoring for epilepsy. J Clin Neurophysiol. 2008;25(3):170-80.

10. Farag S. Stanford-Binet Intelligence test: standardized Arabic version. Cairo, Egypt: Anglo Press; 2011

11. Mueller ST, Piper BJ. The Psychology Experiment Building Language (PEBL) and PEBL test battery. J Neurosci Methods. 2014;222:250-9. https://doi.org/1 0.1016/j.jneumeth.2013.10.024.

12. Monchi $\mathrm{O}$, Petre $\mathrm{V}$, Worsley $\mathrm{K}$, Dagher $\mathrm{A}$. Wisconsin card sorting revisited: distinct neural circuits participating in different stages of the task identified by event-related functional magnetic resonance imaging. J Neurosci. 2001; 21(19):7733-41. https://doi.org/10.1523/JNEUROSCl.21-19-07733.2001.

13. Phillips LH, Wynn VE, McPherson S, Gilhooly KJ. Mental planning and the Tower of London task. Q J Exp Psychol A. 2001;54(2):579-97. https://doi. org/10.1080/713755977.

14. Khalil MS. Preliminary Arabic normative data of neuropsychological tests; the verbal and design fluency. J Clin Experimental Neuropsychol. 2010;32(9): 1028-35. https://doi.org/10.1080/13803391003672305.

15. Bahnasy WS, El-Heneedy YAE, El-Seidy EAS, Ibrahim ISE, Seleem MAH. Primary monosymptomatic nocturnal enuresis: an etiological study. Egypt J Neurol Psychiatr Neurosurg. 2018;45:19.

16. Achenbach TM, Dumenci L. Advances in empirically based assessment: revised cross-informant syndromes and new DSM-oriented scales for the CBCL, YSR, and TRF: comment on Lengua, Sadowksi, Friedrich, and Fischer (2001). J Consult Clin Psychol. 2001;69(4):699-702. https://doi.org/10.1037/ 0022-006X.69.4.699.

17. Marks WN, Zabder NK, Cain SM, Snutch TP, Howland JG. The T-type calcium channel antagonist, Z944, alters social behavior in Genetic Absence Epilepsy Rats from Strasbourg. Behav Brain Res. 2019;361:54-64. https://doi.org/10.1 016/j.bbr.2018.12.021.
18. Caplan R, Siddarth P, Stahl L. Childhood absence epilepsy: behavioral, cognitive, and linguistic comorbidities. Epilepsia. 2008;49(11):1838-46.

19. Vega C, Guo J, Killory B, Danielson N, Vestal M, Berman R, et al. Symptoms of anxiety and depression in childhood absence epilepsy. Epilepsia. 2011; 52(8):e70-4. https://doi.org/10.1111/j.1528-1167.2011.03119.x.

20. Shinnar RC, Shinnar S, Cnaan A, Clark P, Dlugos D, Hirtz DG, et al. Childhood Absence Epilepsy Study Group. Pretreatment behavior and subsequent medication effects in childhood absence epi-lepsy. Neurology. 2017;89(16): 1698-706. https://doi.org/10.1212/WNL.0000000000004514.

21. Masur D, Shinnar S, Cnaan A, Shinnar RC, Clark P, Wang J, et al. Pretreatment cognitive deficits and treatment effects on attention in childhood absence epilepsy. Neurology. 2013;81(18):1572-80 https://doi. org/10.1212/WNL.0b013e3182a9f3ca.

22. Filippini M, Ardu E, Stefanelli S, Boni A, Gobbi G, Benso F. Neuropsychological profile in new-onset benign epilepsy with cen-trotemporal spikes (BECTS): focusing on executive functions. Epilepsy Behav. 2016;54: 71-9. https://doi.org/10.1016/j.yebeh.2015.11.010.

23. Verrotti A, Matricardi S, Rinaldi VE, Prezioso G, Coppola G. Neuropsychological impairment in childhood absence epilepsy: review of the literature. J Neurol Sci. 2015 Dec 15;359(1-2):59-66. https://doi.org/10.1 016/j.jns.2015.10.035.

24. Vindhiya K, Viswanathan V, Balaji P. Clinical profile and neuropsychological assessment of childhood absence epilepsy: a descriptive study. Int J Contemp Pediatr. 2018;5(4):1606-8.

25. Cheng D, Yan X, Gao Z, Xu K, Zhou X, Chen Q. Common and distinctive patterns of cognitive dysfunction in children with benign epilepsy syndromes. Pediatr Neurol. 2017;72:36-41. https://doi.org/10.1016/j.pedia trneurol.2016.12.005

26. Drenthen GS, Fasen F, Fonseca Wald ELA, Backes WH, Aldenkamp AP, Vermeulen RJ, et al. Functional brain network characteristics are associated with epilepsy severity in childhood absence epilepsy. Neurolmage Clin. 2020;27:102264. https://doi.org/10.1016/j.nicl.2020.102264.

27. Cormack F, Cross J, Isaacs E, Harkness W, Wright I, Vargha-Khadem F, et al. The development of intellectual abilities in pediatric temporal lobe epilepsy. Epilepsia. 2007:48(1):201-4. https://doi.org/10.1111/j.1528-1167.2006.00904.x.

28. Sirisamut T, Chinvarun Y, Tantisira MH. Effects of phenytoin and valproic acid on cognitive functions of Thai epileptic patients: a pilot study. J Med Assoc Thail. 2014;97(2):S77.

29. Fonseca Wald ELA, Klinkenberg S, Voncken TPC, Ebus, et al. Cognitive development in absence epilepsy during long-term follow-up. Child Neuropsychol. 2019;25(8):1003-21. https://doi.org/10.1080/09297049.2019.1 614156.

30. Ung H, Cazares C, Nanivadekar A, Kini L, Wagenaar J, Becker D, et al. Interictal epileptiform activity outside the seizure onset zone impacts cognition. Brain. 2017;140(8):2157-68. https://doi.org/10.1093/brain/awx143.

\section{Publisher's Note}

Springer Nature remains neutral with regard to jurisdictional claims in published maps and institutional affiliations.

\section{Submit your manuscript to a SpringerOpen ${ }^{\circ}$ journal and benefit from:}

- Convenient online submission

- Rigorous peer review

- Open access: articles freely available online

- High visibility within the field

- Retaining the copyright to your article

Submit your next manuscript at $>$ springeropen.com 